\title{
NUMERICAL STUDY ON WAVE RUN-UP HEIGHT AND DEPRESSION DEPTH AROUND A VERTICAL CIRCULAR CYLINDER AT VARIOUS FROUDE NUMBERS
}

\author{
Xiaoxi Xiao*, Tahsin Tezdogan and Atilla Incecik \\ Department of Naval Architecture, Ocean and Marine Engineering \\ University of Strathclyde \\ Glasgow, UK \\ *Email: xiaoxi.xiao@strath.ac.uk
}

\begin{abstract}
The turbulent flow past a circular cylinder has been studied extensively by previous researchers due to its importance in many engineering applications. In particular, the wave run-up is one of the most significant design factors when offshore structures are operated. In this paper, the wave run-up height and depression depth around a vertical circular cylinder were numerically investigated. The commercial CFD solver "STAR$\mathrm{CCM}+$ " has been used for the numerical simulations. The models of K-epsilon turbulence and volume of fluid (VOF) are utilised to solve the Reynolds Averaged Navier-Stokes equations (RANS) and continuity equations, respectively. Various Froude numbers and Reynolds numbers are utilised to observe the wave run-up height on the front of the cylinder and the depth of depression at the back. The results were compared with previous experimental data and theoretical values and were found to be in good agreement with other studies.
\end{abstract}

\section{Keywords-Wave Run-up; CFD}

\section{INTRODUCTION}

Several offshore wind farms were built in the last decades to provide green energy to avoid the air pollution. However, there are still some engineering challenges in placing the foundations of offshore wind turbines. In particular, the wave run-up is one of the most significant design factors when offshore structures are operated. The turbulent flow past a circular cylinder has been studied extensively by previous researchers due to its importance in many engineering applications.

There are several experimental and numerical studies on the wave run-up height and depression depth around a surfacepiercing circular cylinder. [1] measured the maximum wave runup heights on the front face of the circular cylinder and the depths of the depression on the centre line behind cylinder at a large range of Froude numbers (Fr) up to about 28 and vertical surface-piercing cylinders of various diameter and draughts were used in the experiments. [2] conducted experiments on a vertical surface-piercing circular cylinder with a large fraught and measured the run-up on the front of the cylinder and the depth of depression at the back. The tests were carried out at a constant ratio of Reynolds number to Froude number of $2.79 \times$ $10^{5}$. Flows past an interface piercing cylinder at various Reynolds and Froude numbers have been simulated by [3] using large eddy simulation (LES) and a volume of fluid (VOF) method. [4] also performed a numerical study of wave run-up height and depression depth around air-water interface-piercing circular cylinder using Computational Fluid Dynamics (CFD) software. They showed that larger wave run-up heights and depression depths are observed as Fr increases. In addition, [5] carried out the experimental study of wave run-up heights on two different shapes of foundation for offshore wind turbines including both regular and irregular waves. A large scale model test investigation on wave run-up in irregular waves at slender piles was performed by [6].

In this paper, the wave run-up height and depression depth around a vertical circular cylinder have been numerically studied by using commercial CFD solver "STAR-CCM+". Various Froude number up to 2.0 and a constant ratio of Reynolds number to Froude number of $2.79 \times 10^{5}$ were used to observe the difference of wave run-up heights and depression depths. The models of K-epsilon turbulence and volume of fluid (VOF) are utilised to solve the Reynolds Averaged Navier-Stokes equations (RANS) and continuity equations, respectively. Additionally, the results are compared with the experimental data published in the literature and theoretical values obtained from Bernoulli's equation.

\section{NUMERICAL MODELLING}

\section{A. Froude numbers and Reynolds numbers selection}

In this paper, various Froude numbers and Reynolds numbers are used. These two non-dimensional numbers are defined as follow:

$$
\begin{aligned}
& F r=\frac{V}{\sqrt{g D}} \\
& R e=\frac{V D}{v}
\end{aligned}
$$


Where, $\mathrm{V}$ is the incoming flow velocity, $\mathrm{g}$ is the gravity acceleration, $\mathrm{D}$ is the diameter of the circular cylinder and $v$ is the kinematic viscosity of the flow.

To compare with the experimental data of [2], there is also a constant ratio of Reynolds number to Froude number of $2.79 \times 10^{5}$ in this paper. The diameter of the cylinder of $0.21 \mathrm{~m}$ that is the same value used in the experiments by [2], gravity acceleration of $9.81 \mathrm{~m} / \mathrm{s}^{2}$ and the dynamic viscosity of $1.078 \times 10^{3}$ $\mathrm{Pa} \cdot \mathrm{s}$ are used in the CFD simulations. Ten different Froude numbers are selected to simulate the wave run-up height and depression depth. The determined Reynolds numbers and flow velocities are shown in Table 1.

Table 1. The selection of Froude number, Reynolds number and corresponding velocities

\begin{tabular}{|c|l|l|l|}
\hline \multicolumn{1}{|l|}{$\begin{array}{l}\text { Case } \\
\text { no. }\end{array}$} & $\begin{array}{l}\text { Froude } \\
\text { number }\end{array}$ & Reynolds & \multicolumn{1}{|l|}{$\begin{array}{l}\text { Flow } \\
\text { number }\end{array}$} \\
\hline 1 & $\mathrm{Fr}=0.2$ & $0.56 \times 10^{5}$ & 0.29 \\
\hline 2 & $\mathrm{Fr}=0.4$ & $1.12 \times 10^{5}$ & 0.57 \\
\hline 3 & $\mathrm{Fr}=0.6$ & $1.67 \times 10^{5}$ & 0.86 \\
\hline 4 & $\mathrm{Fr}=0.8$ & $2.23 \times 10^{5}$ & 1.15 \\
\hline 5 & $\mathrm{Fr}=1.0$ & $2.79 \times 10^{5}$ & 1.44 \\
\hline 6 & $\mathrm{Fr}=1.2$ & $3.35 \times 10^{5}$ & 1.72 \\
\hline 7 & $\mathrm{Fr}=1.4$ & $3.91 \times 10^{5}$ & 2.00 \\
\hline 8 & $\mathrm{Fr}=1.6$ & $4.46 \times 10^{5}$ & 2.30 \\
\hline 9 & $\mathrm{Fr}=1.8$ & $5.02 \times 10^{5}$ & 2.58 \\
\hline 10 & $\mathrm{Fr}=2.0$ & $5.58 \times 10^{5}$ & 2.87 \\
\hline
\end{tabular}

\section{B. Computational Domain}

The computational domain size was set as $-5 \leq \mathrm{x} / \mathrm{D} \leq 25,-8$ $\leq \mathrm{y} / \mathrm{D} \leq 8$, and $-4 \leq \mathrm{z} / \mathrm{D} \leq 2$ for Fr was from 0 to 1.2 . For Fr was from 1.4 to 2.0 , the domain size was $-5 \leq \mathrm{x} / \mathrm{D} \leq 25,-8 \leq \mathrm{y} / \mathrm{D} \leq 8$, and $-4 \leq \mathrm{z} / \mathrm{D} \leq 4$. Larger computational domain was used to simulate the higher wave run-up height at high Froude numbers. Fig. 1. displays the dimensions of the computational domain for large Froude numbers and the top view and side view are both provided.

\section{Mesh Generation}

Mesh generation was carried out by using the automatic mesh model in STAR-CCM+. A trimmed cell mesher was made use of to produce a high-quality grid for complex mesh generation problems. The trimmed mesher is a robust and efficient method and the resulting mesh was formed predominantly of hexahedral cells with trimmed cells next to the surface. Additionally, two volumetric meshes are applied on the near the cylinder structure and air-water interface area in order to refine the meshes as shown in Fig. 2. It is worth noting that 3.6 million cells and 4.2 million cells are applied for two different computational domains, respectively.

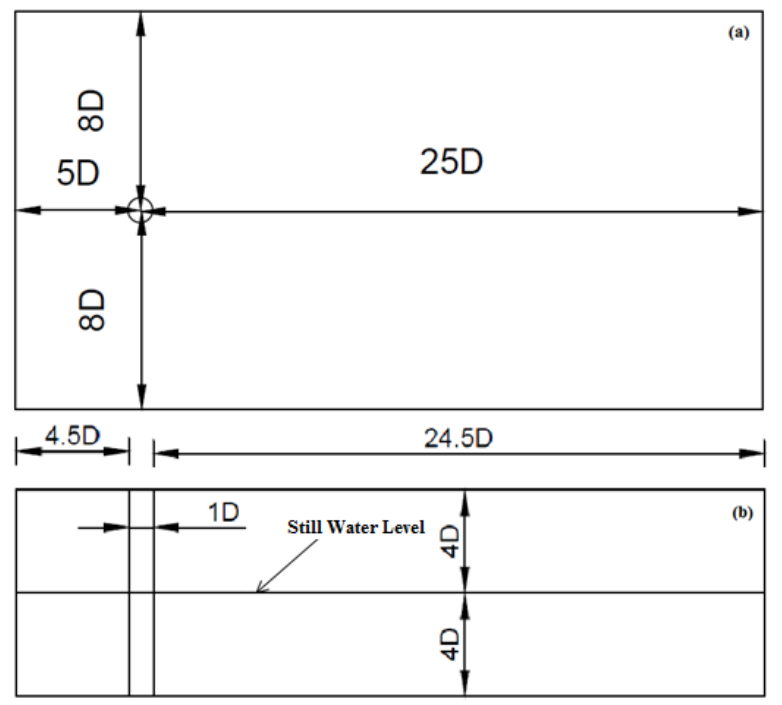

Fig. 1. The dimensions of the computational domain (a) Top View, (b) Side View
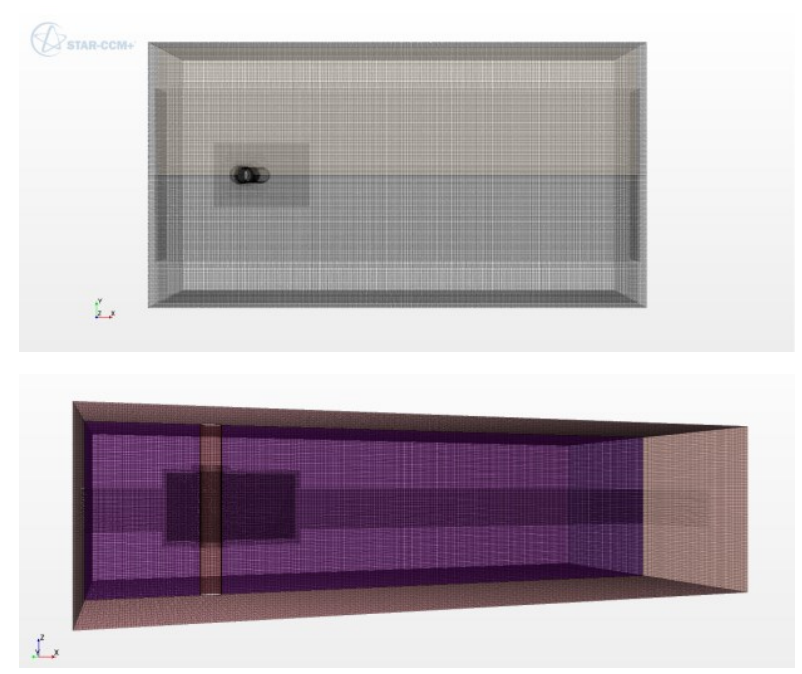

Fig. 2. A general view of the volume mesh inside the computational domain

\section{Boundary Conditions}

In all CFD problems, the initial conditions and boundary conditions must be identified relying on the features of the problem to be solved. The determination of the boundary conditions is extremely significant in order to achieve accurate results. In our study, a velocity inlet boundary condition was set in the positive $\mathrm{x}$ direction where the flat waves were generated and the negative $\mathrm{x}$ direction is modelled as pressure outlet. The both sides, top boundaries were set as velocity inlets. No-slip boundary conditions are applied on the cylinder wall and the bottom. Fig. 3 displays computational domain implemented with all the boundary conditions. Additionally, a damping zone is applied on the outlet boundary condition in order to avoid reflecting waves from outlet boundary. The damping length was half domain size from the cylinder to outlet. 


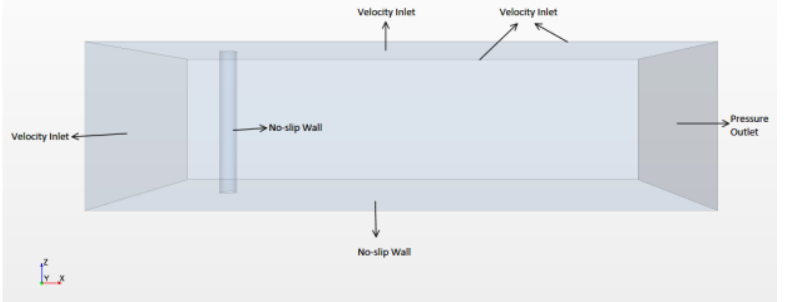

Fig. 3. A general view of computational domain applied with boundary conditions

\section{E. Physics modelling}

The K-epsilon model was selected as the turbulence model in this study and it has become the most widely used model for industrial applications [7]. It is noticed that the K-epsilon model is relatively efficient in terms of CPU time and physical memory, compared to, for example, the Reynolds Stress turbulence model, in which seven equations must be solved in three dimensions as opposed to the two equations of K-epsilon model. For the choice of different K-epsilon turbulence models, the realizable K-epsilon Two-Layer model is selected. The realizable K-epsilon model is substantially better than the standard K-epsilon model for many applications, and can generally be relied upon to give accurate answers. The realizable models have been implemented with a two-layer approach, which activates them to be used with fine meshes that resolve the viscous sublayer and high Reynolds number versions. A K-epsilon turbulence model is a two-equation model in which transport equations are solved for the turbulent kinetic energy $\mathrm{k}$ and its dissipation rate $\varepsilon$. This model contains a new transport equation for the turbulent dissipation rate $\varepsilon$ [8]. Also, a critical coefficient of the model, $C_{\mu}$, is expressed as a function of mean flow and turbulence properties, rather than assumed to be constant as in the standard model. This procedure lets the model satisfy certain mathematical constraints on the normal stresses consistent with the physics of turbulence. The concept of a variable $C_{\mu}$ is also consistent with experimental observations in boundary layers.

The transport equations for the realizable K-epsilon model are:

$$
\begin{gathered}
\frac{d}{d t} \int_{V} \rho k d V+\int_{A} \rho k\left(\mathbf{v}-\mathbf{v}_{g}\right) \cdot d \mathbf{a}= \\
\int_{A}\left(\mu+\frac{\mu_{t}}{\sigma_{k}}\right) \nabla k \cdot d \mathbf{a}+\int_{V^{2}}\left[f_{C} G_{k}+G_{b}-\rho\left(\left(\varepsilon-\varepsilon_{0}\right)+Y_{M}\right)+S_{k}\right] d V \\
\frac{d}{d t} \int \rho \varepsilon d V+\int_{V} \rho \varepsilon\left(\mathbf{v}-\mathbf{v}_{g}\right) \cdot d \mathbf{a}= \\
\int_{A}\left(\mu+\frac{\mu_{t}}{\sigma_{\varepsilon}}\right) \nabla \varepsilon \cdot d \mathbf{a}+\int_{V}\left[f_{C} C_{\varepsilon_{1}} S \varepsilon+\frac{\varepsilon}{k}\left(C_{\hat{\vartheta}_{1}} C_{\varepsilon_{3}} G_{b}\right)-\frac{\varepsilon}{k+\sqrt{v \varepsilon}} C_{\varepsilon} \rho\left(\varepsilon-\varepsilon_{0}\right)+S_{\varepsilon}\right] d V
\end{gathered}
$$

where:

\section{$\rho$ is the fluid density}

$\mathrm{k}$ is the the turbulent kinetic energy.

$\varepsilon$ is turbulent dissipation rate
$S_{k}$ and $S_{\varepsilon}$ are the user-specified source terms.

$\varepsilon_{0}$ is the ambient turbulence value in the source terms that counteracts turbulence decay.

$f_{c}$ is the curvature correction factor.

The turbulent viscosity $\mu \_t$ is computed as:

$$
\mu_{t}=\rho C_{\mu} \frac{k^{2}}{\varepsilon}
$$

where the coefficient $C_{\mu}$ is no longer constant as with the standard K-Epsilon model, but is determined:

$$
C_{\mu}=\frac{1}{A_{0}+A_{s} U^{(*)} \frac{k}{\varepsilon}}
$$

The Volume of Fluid method is a simple multiphase model that is used to resolve the interface between the phases of the mixture. Due to the numerical efficiency of this method, the model is suited for simulations of flows where each phase forms a large structure, with a relatively small total contact area between the different phases. A good example of this type of flow is sloshing flow in a water tank, where the free surface always remains smooth. If the movement of the tank becomes pronounced, breaking waves, large numbers of air bubbles in the water, and water droplets in the air will appears. The method would then require a finer mesh to gain more accurate results. Water and air are considered as the two immiscible components in our study and a value of 0.5 for the volume fraction of water indicated that a computational cell is filled with $50 \%$ water and $50 \%$ air. This value, therefore, indicates the position of the airwater interface, which corresponds to the free surface. It is shown in Fig.4 that the free surface was represented in this CFD model by display the water volume fraction.

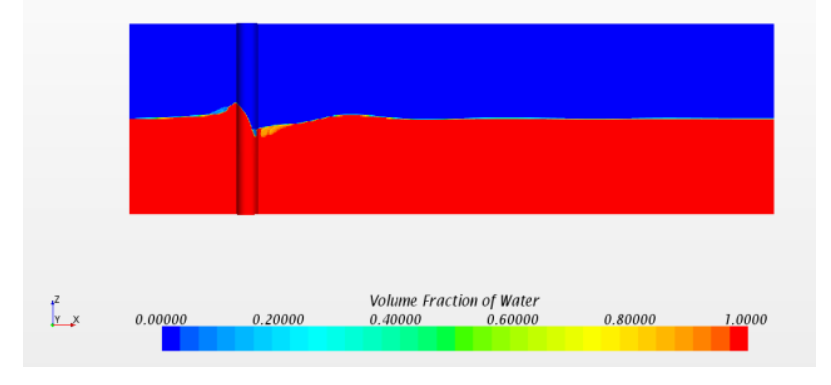

Fig. 4. Free surface representation on the centreline domain face

\section{F. Time-step selection}

The Courant number (CFL) is the ratio of the physical time step to the mesh convection time scale, relates the mesh cell dimension to the mesh flow speed $U$.

$$
C F L=\frac{U \Delta t}{\Delta x}
$$

The Courant number is generally calculated for each cell be less than or equal to 1 for numerical stability. If the Courant number is $<=1$ then the fluid particles move from one cell to another within one time step (at most). While if the Courant number is $>1$ a fluid particle moves through two or more cells at each time step and this can affect convergence negatively. In 
order to obtain relatively accurate results within a reasonable running time, the time size is determined by $\Delta t=0.005 * V / D$ according to the guidelines of [9]. In addition, a first-order temporal scheme was applied to discretise the unsteady term in the Navier-Stokes equations.

\section{RESULTS AND DISCUSSIONS}

The following section will present not only the simulation results obtained during this study but also the comparison with experimental results published in the literature and theoretical values obtained from Bernoulli's equation.

\section{A. Flow near the cylinder structure}

The following Fig.5 and Fig. 6 illustrate the air-water interface around the circular cylinder for different Froude Numbers for front view and side view respectively. At smallest $\mathrm{Fr}=0.2$, we can find that the deformation of the free surface is insignificantly small. At $\mathrm{Fr}=0.4$, the elevation of free surface increases but it is still very small. For $\mathrm{Fr}=0.6 \sim 0.8$, relative smaller bow waves are observed in the front of the vertical cylinder and the Kelvin waves are behind the cylinder. For $\mathrm{Fr}=1.0 \sim 1.4$, much increased bow waves are observed in front of the cylinder and depression appears on the downstream side of the cylinder. For Fr=1.6 1.8, the bow wave rises notably and plunging breaking wave is observed around the cylinder. At the highest $\mathrm{Fr}=2.0$, the bow wave reaches the maximum elevation and it is much higher than those with other Fr.

(a)

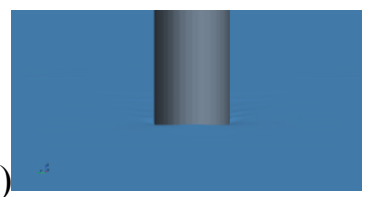

(c)

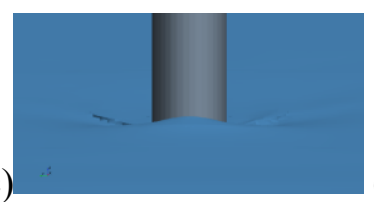

(e)

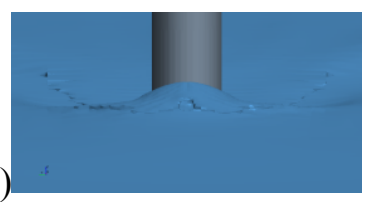

(g)

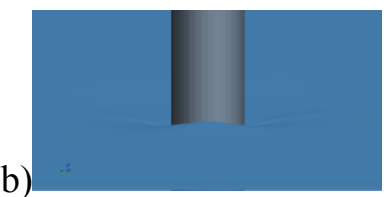

(d)

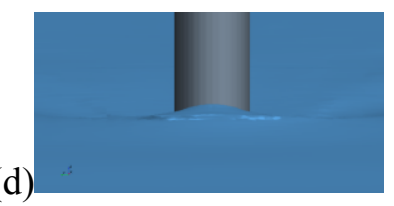

(f)

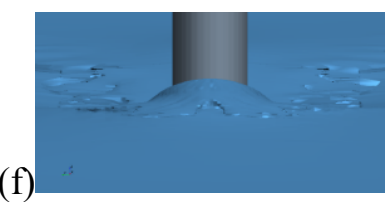

(h)

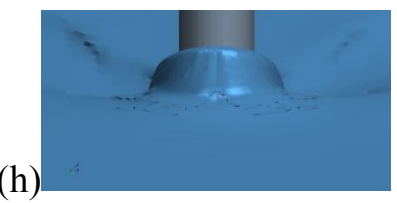

(i)
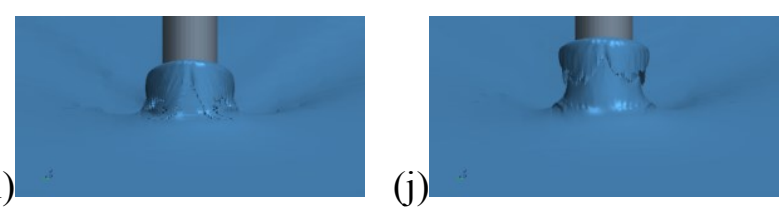

Fig. 5. Front view of flow past vertical cylinder for different Froude Numbers (a) $\mathrm{Fr}=0.2$, (b) $\mathrm{Fr}=0.4$, (c) $\mathrm{Fr}=0.6$, (d) $\mathrm{Fr}=0.8$, (e) $\mathrm{Fr}=1.0$, (f) $\mathrm{Fr}=1.2$, (g) $\mathrm{Fr}=1.4$, (h) $\mathrm{Fr}=1.6$, (i) $\mathrm{Fr}=1.8$, (j) $\mathrm{Fr}=2.0$
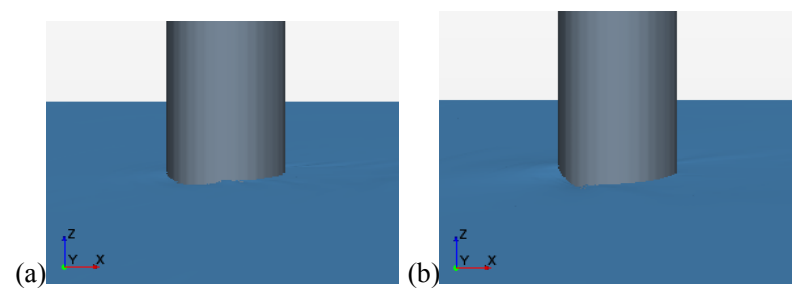

(c)
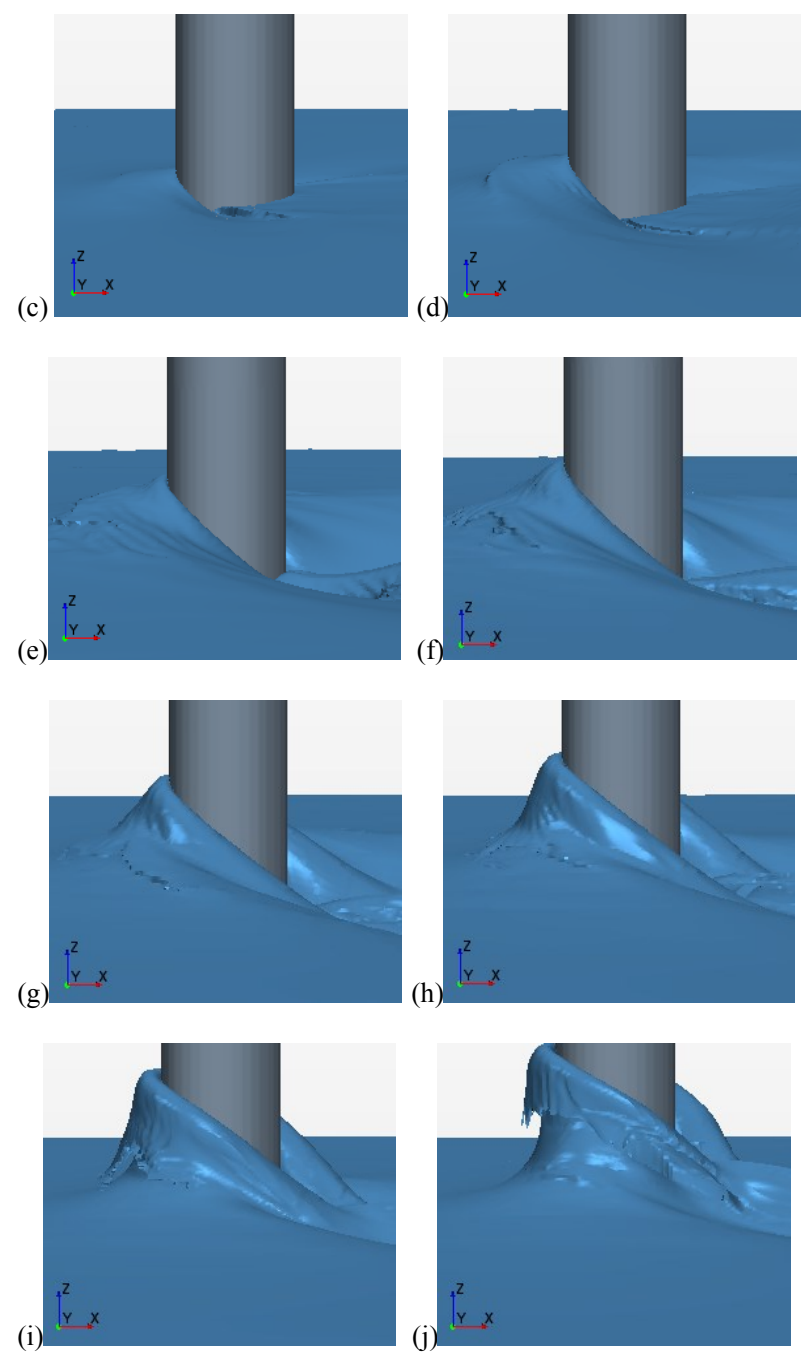

Fig. 6. Side view of flow past vertical cylinder for different Froude Numbers (a) $\mathrm{Fr}=0.2$, (b) $\mathrm{Fr}=0.4$, (c) $\mathrm{Fr}=0.6$, (d) $\mathrm{Fr}=0.8$, (e) $\mathrm{Fr}=1.0$, (f) $\mathrm{Fr}=1.2$, (g) $\mathrm{Fr}=1.4$, (h) $\mathrm{Fr}=1.6$, (i) $\mathrm{Fr}=1.8$, (j) $\mathrm{Fr}=2.0$

\section{B. Wave run-up height and depression depth}

The wave run-up height is the maximum bow wave height in front of the cylinder and the depression depth is the largest cavity depth behind the cylinder. The free surface elevation contours that indicate the run-up height and depression depth with different Fr numbers are shown in Fig.7. As we can see that, both wave run-up height and depression depth increase as Fr increases except that at $F r=2.0$, the depression depth is around $0.05 \mathrm{~m}$ which is much smaller than the depression depth of $\mathrm{Fr}=1.8$. 
(a)
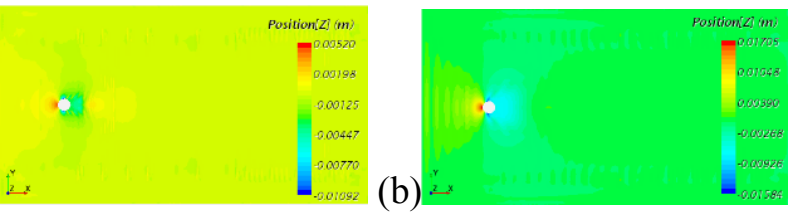

(c)
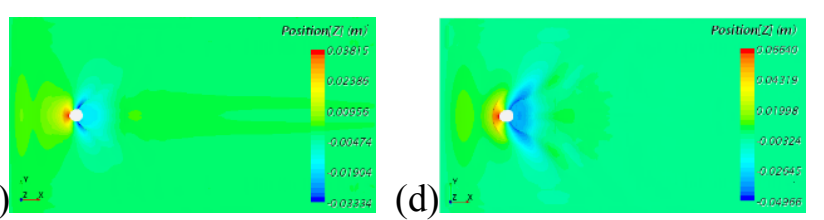

(e)
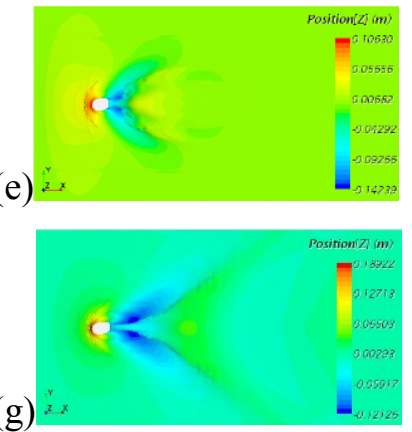

(f)
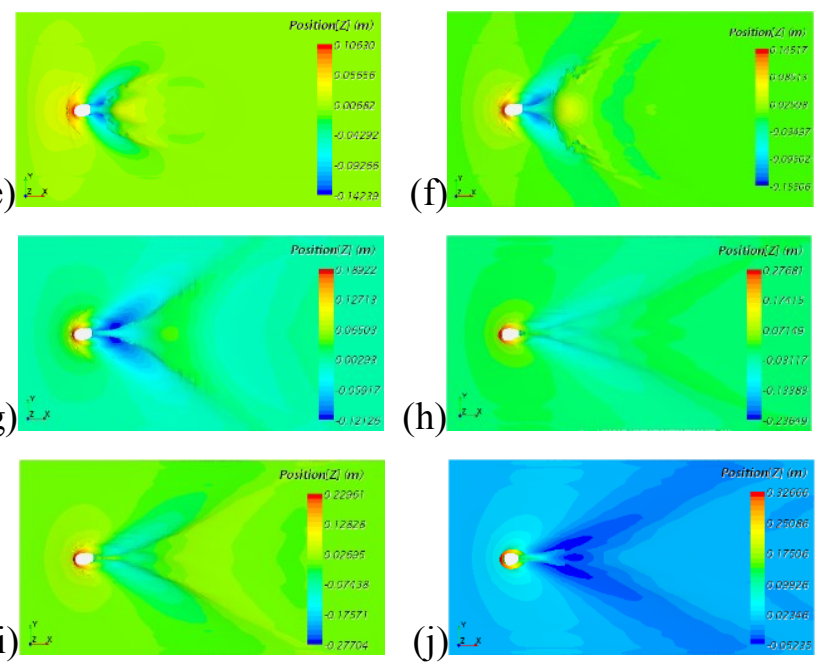

Fig. 7. Free surface elevation contours for different Froude Numbers (a) $\mathrm{Fr}=0.2$, (b) $\mathrm{Fr}=0.4$, (c) $\mathrm{Fr}=0.6$, (d) $\mathrm{Fr}=0.8$, (e) $\mathrm{Fr}=1.0$, (f) $\mathrm{Fr}=1.2$, (g) $\mathrm{Fr}=1.4$, (h) $\mathrm{Fr}=1.6$, (i) $\mathrm{Fr}=1.8$, (j) $\mathrm{Fr}=2.0$

\section{Comparison with the experimental data and theoretical values}

The wave run-up results of CFD simulations and results from Bernoulli's equation, $\mathrm{D} 1 / \mathrm{d}=\mathrm{Fr}^{2} / 2$ (D1 is the wave run-up height and $\mathrm{d}$ is the cylinder diameter), are presented in Table 2 . It is observed that when the Fr number is low, the simulation results are in good agreement with the theoretical values. When the $\mathrm{Fr}$ number is larger than 1.0, the simulation results are becoming slightly lower than the theoretical values. When the Fr number increases from 1.6 to 2.0 , the theoretical values are becoming much larger than the simulation results. However, the trend is constantly the same that the wave run-up height increases as the Fr number increases. In Fig. 8, the simulation results and the measurements of the experiment of [2] are plotted with Bernoulli's equation. The results from CFD simulations is consistent with the measurements of [2]. It can be seen that the trend is the same that the Fr number at which D1/d first falls below $\mathrm{Fr}^{2} / 2$ is between 1 and 1.6. As we can see that the results from CFD simulations and the experiment data are less than the values of Bernoulli's equation, it is because that the Bernoulli's equation neglects the viscosity and in a viscous flow, energy is dissipated due to the viscous forces. Moreover, the depression depth is very small at $\mathrm{Fr}=2.0$ which is different from the result of [1]. It is due to that when at high Froude number of 2.0, the vortices appear behind the cylinder which affect the deformation of water surface. Another possible solution is that finer mesh is required around the cylinder structure.

Table 2. Comparison of wave run up height results from simulations and Bernoulli's equation

\begin{tabular}{|c|c|c|c|}
\hline $\begin{array}{l}\text { Froude } \\
\text { Number }\end{array}$ & $\begin{array}{c}\text { Wave } \\
\text { Run-up } \\
\text { Height (m) }\end{array}$ & $\begin{array}{c}\text { Wave Run- } \\
\text { up height } \\
\text { to cylinder } \\
\text { diameter }\end{array}$ & $\begin{array}{c}\text { Bernoulli's } \\
\text { equation } \\
\mathrm{Fr}^{2} / 2\end{array}$ \\
\hline 0.2 & 0.005 & 0.024 & 0.02 \\
\hline 0.4 & 0.017 & 0.081 & 0.08 \\
\hline 0.6 & 0.038 & 0.181 & 0.18 \\
\hline 0.8 & 0.068 & 0.324 & 0.32 \\
\hline 1.0 & 0.104 & 0.495 & 0.50 \\
\hline 1.2 & 0.146 & 0.695 & 0.72 \\
\hline 1.4 & 0.189 & 0.900 & 0.98 \\
\hline 1.6 & 0.229 & 1.090 & 1.28 \\
\hline 1.8 & 0.277 & 1.319 & 1.62 \\
\hline 2.0 & 0.327 & 1.557 & 2.00 \\
\hline
\end{tabular}

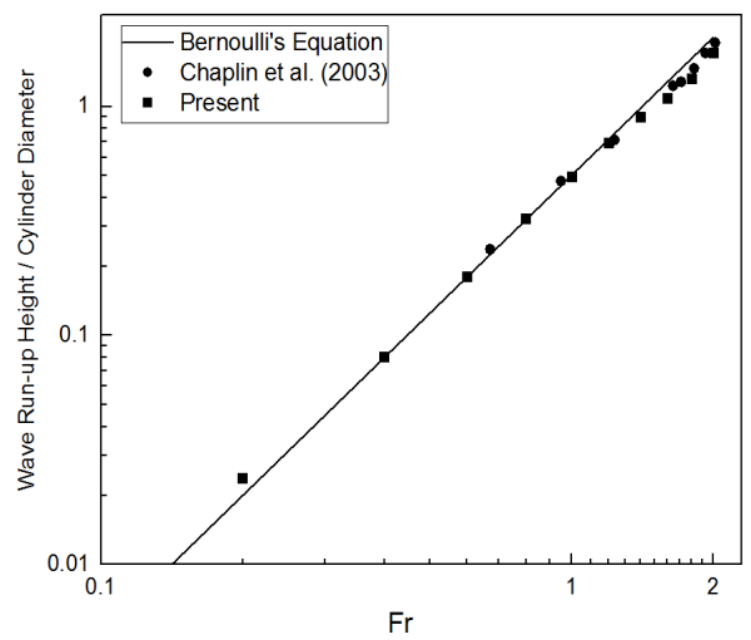

Fig. 8. Comparison of CFD simulation results, theoretical values and experimental measurements of [2]

\section{SUMMARY AND CONCLUSIONS}

The wave run-up height and depression depth around a vertical circular cylinder have been investigated by using the commercial CFD solver "STAR-CCM+" version 10.04. In addition, a constant ratio of Reynolds number to Froude number of $2.79 \times 10^{5}$ is utilised in this paper. Various Froude numbers from 0.2 to 2.0 are applied to study the wave run up height on 
the front of the cylinder and depression depth at the rear. The models of K-epsilon turbulence and volume of fluid (VOF) are utilised to solve the Reynolds Averaged Navier-Stokes equations (RANS) and continuity equations, respectively. Moreover, the mesh generation study and the selections of time step and boundary conditions are presented. The CFD simulation results are compared with both the measurements of [2] and the theoretical values of Bernoulli's equation and it shows that the results are in good agreement. It can be seen that wave run-up height and depression depth increases as the Fr number increases. However, at high Fr numbers, from 1.0 to 2.0, the CFD simulation results are lower than the theoretical values. At the largest $\mathrm{Fr}=2.0$, the simulation results are lower than theoretical values by approximately $33 \%$. It is because that the Bernoulli's equation neglects the viscosity and the viscous forces result in the energy dissipation especially at large Froude numbers which is also with large velocities and Reynolds numbers. Finer mesh is required to obtain accuracy results for high Fr number simulations. The relative lower depression depth is observed at $\mathrm{Fr}=2.0$ and it calls for further work to simulate the depression depth with high Fr numbers.

\section{REFERENCES}

[1] Hey, A. D., Flow about Semi-Submerged Cylinders of Finite Length, Princeton University Report. 1947

[2] Chaplin, J. R. and P. Teigen, "Steady Flow past a Vertical SurfacePiercing Circular Cylinder. Journal of Fluids and Structures", Vol. 18 , pp. 271-285. 2003

[3] Yu, G., E. J. Avital and J. J. R. Williams, "Large Eddy Simulation of Flow Past Free Surface Piercing Circular Cylinders". Journal of Fluids Engineering, Vol. 130, pp. 101304.1-101304.9. 2008

[4] Koo, B. G., Park, D. W. and Paik, K. J., "A Study on Wave Run-up Height and Depression Depth around Air-water Interface-piercing Circular Cylinder". Journal of the Korean Society of Marine Environment \& Safety, Vol. 20, No. 3, pp. 312-317. 2014

[5] De Vos, L., Frigaard, P., De Rouck, J. "Wave run-up on cylindrical and cone shaped foundations for offshore wind turbines". Coastal Engineering 54 (1), 17-29. 2007

[6] Ramirez. J., Frigaard. P., Lykke Andersen. T., De Vos. L. (2013) Large scale model test investigation on wave run-up I in irregular waves at slender piles. Ocean Engineering 72 (2013) 69-79.

[7] CD-adapco, User Guide STAR-CCM+ Version 10.04.009. 2014

[8] Shih, T.-H., Liou, W.W., Shabbir, A., Yang, Z. and Zhu, J. (1994). “A New k- Eddy Viscosity Model for High Reynolds Number Turbulent Flows -- Model Development and Validation”, NASA TM 106721.

[9] International Towing Tank Conference (ITTC), 2011b. Practical guidelines for ship CFD applications. In :Proceedings of the $26^{\text {th }}$ ITTC. 